\title{
Multi-material 3D printed mechanical metamaterials: Rational design of elastic properties through spatial distribution of hard and soft phases
}

Cite as: Appl. Phys. Lett. 113, 241903 (2018); https://doi.org/10.1063/1.5064864

Submitted: 09 October 2018 . Accepted: 27 November 2018 . Published Online: 14 December 2018

M. J. Mirzaali (D), A. Caracciolo, H. Pahlavani, S. Janbaz (D, L. Vergani, and A. A. Zadpoor
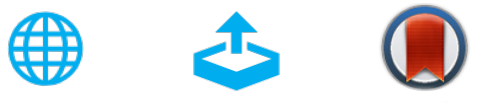

\section{ARTICLES YOU MAY BE INTERESTED IN}

Compact acoustic double negative metamaterial based on coexisting local resonances Applied Physics Letters 113, 244101 (2018); https://doi.org/10.1063/1.5052026

Maximizing the quality factor to mode volume ratio for ultra-small photonic crystal cavities Applied Physics Letters 113, 241101 (2018); https://doi.org/10.1063/1.5064468

Acoustic perfect absorbers via spiral metasurfaces with embedded apertures Applied Physics Letters 113, 233501 (2018); https://doi.org/10.1063/1.5063289

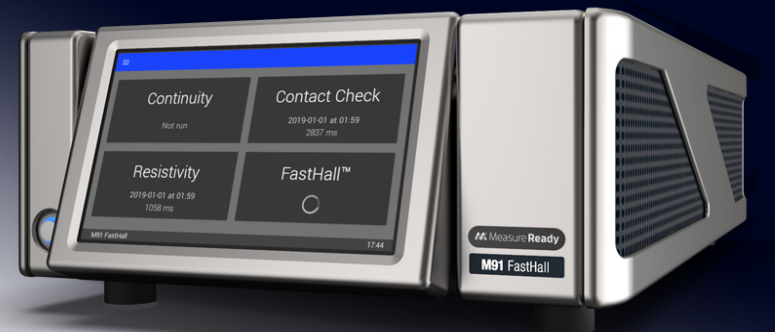

M Measure Ready M91 FastHall ${ }^{\mathrm{TM}}$ Controller

A revolutionary new instrument for complete Hall analysis 


\title{
Multi-material 3D printed mechanical metamaterials: Rational design of elastic properties through spatial distribution of hard and soft phases
}

\author{
M. J. Mirzaali, ${ }^{1, a)}$ A. Caracciolo, ${ }^{2}$ H. Pahlavani, ${ }^{1}$ S. Janbaz, ${ }^{1}$ L. Vergani, ${ }^{2}$ and A. A. Zadpoor ${ }^{1}$ \\ ${ }^{1}$ Department of Biomechanical Engineering, Faculty of Mechanical, Maritime, and Materials Engineering, \\ Delft University of Technology (TU Delft), Mekelweg 2, 2628 CD Delft, The Netherlands \\ ${ }^{2}$ Department of Mechanical Engineering, Politecnico di Milano, via La Masa, 1, 20156 Milano, Italy
}

(Received 9 October 2018; accepted 27 November 2018; published online 14 December 2018)

\begin{abstract}
Up until recently, the rational design of mechanical metamaterials has usually involved devising geometrical arrangements of micro-architectures that deliver unusual properties on the macroscale. A less explored route to rational design is spatially distributing materials with different properties within lattice structures to achieve the desired mechanical properties. Here, we used computational models and advanced multi-material 3D printing techniques to rationally design and additively manufacture multi-material cellular solids for which the elastic modulus and Poisson's ratio could be independently tailored in different (anisotropic) directions. The random assignment of a hard phase to originally soft cellular structures with an auxetic, zero Poisson's ratio, and conventional designs allowed us to cover broad regions of the elastic modulus-Poisson's ratio plane. Patterned designs of the hard phase were also used and were found to be effective in the independent tuning of the elastic properties. Close inspection of the strain distributions associated with the different types of material distributions suggests that locally deflected patterns of deformation flow and strain localizations are the main underlying mechanisms driving the above-mentioned adjustments in the mechanical properties. Published by AIP Publishing.

https://doi.org/10.1063/1.5064864
\end{abstract}

Mechanical metamaterials are designer materials with unusual properties that originate from their rational design at the micro-scale. ${ }^{1-3}$ Some examples of these unusual properties are negative Poisson's ratios ${ }^{4}$ and bistability, ${ }^{5}$ shape-morphing mechanical metamaterials, ${ }^{6}$ negative compressibility, ${ }^{7}$ negative stiffness, ${ }^{8}$ crumpled metamaterials, ${ }^{9}$ and tunable negative thermal expansion. ${ }^{10}$

The vast majority of the mechanical metamaterials presented to date including most of the above-mentioned examples have used various types of geometrical designs to achieve their unusual properties. However, the rational design of mechanical metamaterials is not limited to their small-scale geometry and includes rationally designing the spatial distribution of the mechanical properties as well as a combination with a geometrical design and spatial distribution of mechanical properties. The research into both latter rational design approaches has just started, as the multimaterial 3D printing (= additive manufacturing) techniques required for achieving complex spatial distributions of mechanical properties and combining that with complex geometries are just emerging. A few recent studies on 3D lattices with high Poisson's ratio properties, ${ }^{11}$ topology optimization of multi-material mechanical metamaterials with negative Poisson's ratio ${ }^{12}$ or multifunctionality, ${ }^{13}$ and controlling instabilities, ${ }^{14}$ are examples of the applications of dual-phase materials ${ }^{15,16}$ for achieving new ranges of properties and new types of functionalities in mechanical metamaterials.

\footnotetext{
${ }^{\text {a) }}$ Author to whom correspondence should be addressed: M.J.Mirzaali Mazandarani@tudelft.nl. Tel.: +31-15-2783133.
}

An important aim in the design of mechanical metamaterials is independent tailoring of the elastic modulus and Poisson's ratio. ${ }^{17}$ Adjusting the Poisson's ratio of mechanical metamaterials in a wide range of negative and positive values allows for devising a rich set of new functionalities. For example, negative values of Poisson's ratios (i.e., auxetic mechanical metamaterials ${ }^{2}$ ) could be combined with positive values (i.e., conventional mechanical metamaterials) to design orthopaedic implants with improved longevity ${ }^{18}$ and to enable complex local actuations in soft robotics using a single far-field force. ${ }^{19}$ At the same time, tailoring the stiffness values of mechanical metamaterials allows for adjustment of their load-bearing capability and compliancy. For example, mechanical metamaterials with extremely high negative or positive Poisson's ratios often lack high elastic moduli. Rational design approaches are therefore required to increase (decrease) the elastic modulus regardless of the Poisson's ratio of the metamaterial. Here, we use complex spatial distributions of the mechanical properties realized through advanced multi-material $3 \mathrm{D}$ printing processes and combine that with architectured geometrical designs to independently tailor the Poisson's ratio and elastic modulus of dual-phase (i.e., soft-hard) mechanical metamaterials.

We used computational models for the rational design of the mechanical metamaterials. A large number of the designed specimens were then fabricated and were subjected to mechanical loading to characterize their mechanical behavior. In our designs, we used three unit cells, representing lattice structures with negative $\left(\theta=60^{\circ}\right)$, intermediate (i.e., zero) $\left(\theta=90^{\circ}\right)$, and positive $\left(\theta=120^{\circ}\right)$ Poisson's ratios [Fig. 1(a)]. The unit cells had a similar height $(c)$ and 
(a)
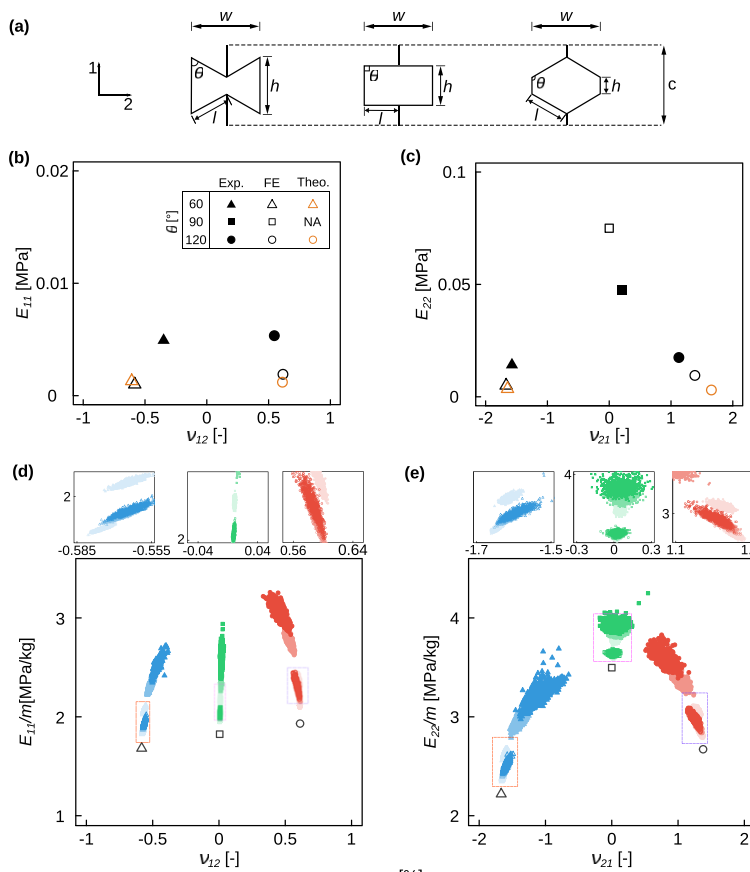

(e)
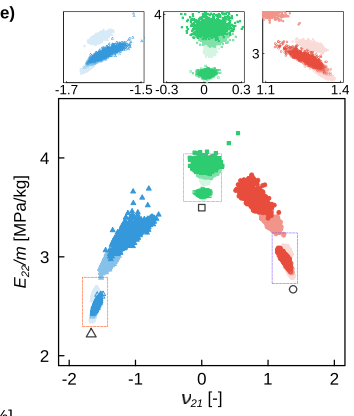

$\rho_{h}[\%$

\begin{tabular}{|c|c|c|c|c|c|c|c|}
\hline \multirow[b]{3}{*}{$E_{h} / E_{s}[-]$} & \multicolumn{7}{|c|}{$\rho_{h}[\%]$} \\
\hline & 0 & \multicolumn{3}{|c|}{25} & \multicolumn{3}{|c|}{50} \\
\hline & 1 & 10 & 100 & 1000 & 10 & 100 & 1000 \\
\hline \multirow{3}{*}{ ప } & $\Delta$ & & $\Delta$ & $\Delta$ & $\Delta$ & $\Delta$ & $\Delta$ \\
\hline & 口 & & ㅁ & व & & " & - \\
\hline & 。 & & ० & 0 & 。 & • & $\bullet$ \\
\hline
\end{tabular}

FIG. 1. (a) Three unit cell geometries $\left(\theta=60^{\circ}, 90^{\circ}\right.$, and $\left.120^{\circ}\right)$ used for the fabrication of lattice structures. A comparison of the numerical results, experimental obervations, and theoretical predictions for the lattice structures made from a uniform (soft) material and tested in directions 1 (b) and 2 (c). The regions covered by the mechanical porperties of multi-material mechanical metamaterials with three geometries and random assigment of a hard phase to the elements of the lattice structure until two fractions of the hard material, $\rho_{h}=25 \%$ and $50 \%$, were achieved. Moreover, three different values of $\frac{E_{h}}{E_{s}}$ were used to calculate the elastic modulus and Poisson's ratio in directions 1 (d) and 2 (e). The specific elastic stiffnesses, i.e., normalized by the mass, $m$, of the sample are presented in (d) and (e).

width (w) while differing in $h$ and $l$ [Fig. 1(a)]. Keeping a specific set of design parameters constant allowed us to design lattice structures with an equal overall width $(W)$ and length $(L)$. The in-plane $(t)$ and out-of-plane thicknesses $(b)$ of the unit cells with different angles were similar as well. All design parameters are listed in the supplementary material (Table S1).

To create the computational models, the geometry of each lattice structure was created as an input file in Matlab (R2011b) and later was handled with the finite element modeling software (Abaqus 6.14). In-plane Timoshenko beam elements (B21, Abaqus) with a rectangular crosssection were used to model the lattice structures. A hyperelastic material model (Neo Hookean, $C_{10}=0.106 \mathrm{MPa}$, $D_{1}=0.03 \mathrm{MPa}^{-1}$ ) was applied as the constitutive equation of the soft phase. Three levels of the elastic modulus of the hard phase were selected with one, two, or three orders of magnitude higher elastic moduli as compared to the soft phase (i.e., $\frac{E_{h}}{E_{s}}=10,100$, or 1000). Furthermore, two levels of the fraction of the hard phase with respect to the overal volume (i.e., $\rho_{h}=25 \%$ and $50 \%$ ) were chosen when spatially distributing both phases in the lattice strucutres.

A large number of finite element models of the lattice structures were created in which the hard phase replaced the soft elements of the lattice structures at random locations. The replacement of the soft phase with the hard phase continued until the intended fraction of the hard phase was achieved. To adequately sample the stochastic space of possible designs, one thousand simulations were performed for each combination of the hard phase fraction and $\frac{E_{h}}{E_{\mathrm{s}}}$, resulting in a total of 18000 simulations.

Moreover, the simulations were performed in two directions, i.e., 1 and 2, in order to investigate the anisotropic behavior of these metamaterials. A displacement boundary condition equal to $10 \%$ longitudinal/transverse strain was applied to simulate a tensile test. The boundary conditions were applied to a reference point, which was kinematically coupled to the corresponding nodes of the lattice structures. For instance, for the simulation in the 1-direction [Fig. 1(a)], a displacement was applied on a top reference point. Another reference node was created at the bottom of the structure, which was clamped. A non-linear static simulation was performed. The stresses $\left(\sigma_{11}, \sigma_{22}\right)$ and strains $\left(\varepsilon_{11}, \varepsilon_{22}\right)$ in both directions were, respectively, calculated based on the ratio of the reaction force to the cross section area and the ratio of the displacement to the free length of the structure in the longitudinal (transverse) direction. The elastic stiffnesses $\left(E_{11}, E_{22}\right)$ of the structure was calculated as the slope of the stress-strain curve at $1 \%$ strain.

The Poisson's ratio in direction 1 was calculated as $\nu_{12}=-\frac{\varepsilon_{22}}{\varepsilon_{11}}$, where $\varepsilon_{11}$ is the applied strain in direction 1 , and $\varepsilon_{22}$ is the sum of the transverse deformation of the structure under this displacement at every $i$ th node $\left(U_{22, i}\right)$ with $\varepsilon_{22}=\frac{\sum_{i=1}^{n} U_{22, i}}{W}$ and $n=$ total number of lateral nodes along direction 2. Likewise, the Poisson's ratio in direction 2 was defined as $\nu_{21}=-\frac{\varepsilon_{11}}{\varepsilon_{22}}$, where $\varepsilon_{22}$ is the applied strain in direction 2 and $\varepsilon_{11}=\frac{\sum_{i=1}^{p} U_{11, i}}{L}$ with $p$ equal to the total number of the lateral nodes in direction 1. The Poisson's ratios in both directions were measured at $1 \%$ longitudinal (transverse) strain.

For the experimental study, fifteen specimens were directly fabricated using a multi-material 3D printer (Object500 Connex3 3D printer, Stratasys) working on the basis material jetting (polyjet technology). Of those specimens, 6 and 9 samples were tested in directions 1 and 2, respectively. Among the specimens mentioned above, 5 were purely soft while the rest were multi-material. The hard and soft phases were respectively printed with VeroCyan [RGD841, shore hardness (D) 83-86] and Agilus30 Black [FLX985, shore hardness (A) 30-35]. These materials were selected to achieve $\frac{E_{h}}{E_{s}}$ values of up to $\approx 1000$.

The specimens tested in direction 1 had an extra hard part to make it possible to attach it to the gripper while the ones tested in direction 2 were directly attached to the gripper via pins. Two gripping systems and their pins were designed and additively manufactured using a fused deposition modeling (FDM) 3D printer (Ultimaker 2+, Geldermalsen, The Netherlands) from polylactic acid (PLA) filaments (MakerPoint PLA 750 gr Natural).

Tensile mechanical testing was performed under displacement control using an LLOYD instrument (LR5K) mechanical testing machine with a $100 \mathrm{~N}$ load cell and a 
stroke rate of $2 \mathrm{~mm} / \mathrm{min}$. The time, force, and displacement were recorded at a sampling rate of $20 \mathrm{~Hz}$. The force and displacement were used to calculate the stress and strain with respect to the initial cross-section area and the initial free length of the specimens. The stiffness of the structure was determined using the measured stress and strain values. The deformation of the specimens was also captured by a digital camera that was later used to calculate the Poisson's ratios in both directions using image analysis (see the supplementary material, Fig. S1).

We validated the accuracy of our numerical simulations for the purely soft lattice structures by comparing them with the experimental and theoretical models [Fig. 1(b)]. Equation (1) shows the existing theoretical relations for the calculation of the elastic properties of homogenized lattice structures $^{20}$

$$
\begin{aligned}
& E_{11}=\frac{k_{f}(h / l+\sin \theta)}{b \cos ^{3} \theta}, \quad E_{22}=\frac{k_{f} \cos \theta}{b(h / l+\sin \theta) \sin \theta}, \\
& \nu_{12}=\frac{\sin \theta(h / l+\sin \theta)}{\cos ^{2} \theta}, \quad \nu_{21}=\frac{\cos ^{2} \theta}{\sin \theta(h / l+\sin \theta)},
\end{aligned}
$$

where $k_{f}=E_{b} b(t / l)^{3}$ is the flexure force constant and $E_{b}$ is the elastic modulus of the solid constituent. The elastic modulus, $E_{s}$, of our soft materials is $0.6 \mathrm{MPa}$.

We found that the elastic properties obtained from our computational models for the homogenously soft materials are within the range of those calculated using Eq. (1) (Theo.) and experimental observations (Exp.) [Figs. 1(b) and 1(c)].

The random assignment of the hard phase to the elements of the lattice structures considered here resulted in drastic changes in both the Poisson's ratio and elastic modulus [Figs. 1(d) and 1(e)]. Specific regions, therefore, emerged within the elastic modulus-Poisson's ratio plane within which the Poisson's ratio and elastic modulus could be independently adjusted [Figs. 1(d) and 1(e)]. Generally speaking, the changes in both Poisson's ratio and elastic modulus were larger in direction 2 as compared to direction 1 [Figs. 1(d), 1(e), and 2]. Moreover, the elastic properties of lattice structures with $\theta=60^{\circ}$ and $\theta=120^{\circ}$ were much more sensitive to the presence of a second, harder phase as compared to the lattice strucutres with $\theta=90^{\circ}$ [Figs. 1(d) and 1(e)]. This trend was particularly clear for the Poisson's ratio in direction 1 and elastic modulus in direction 2 [Figs. 1(d) and 1(e)]. Random assignment of a hard phase $\left(\frac{E_{h}}{E_{s}}=10-1000\right)$ to up to $50 \%$ of the elements of the considered lattice strucutres resulted in up to $\approx 2$ orders of magnitude increase in the elastic modulus [Figs. 1(d) and 1(e)]. Combining the hard and soft phases shifted the Poisson's ratio of the lattice strucutres towards higher values in the case of $\theta=60^{\circ}$ and towards lower values in the case of $\theta=120^{\circ}$ [Figs. 1(d), 1(e), and 2(a)-2(d)]. The distribution of the Poisson's ratio of the original value (i.e., the Poisson's ratio of a single-material lattice strucutre) was more or less symmetric in the case of $\theta=90^{\circ}$ [Figs. 1(d) and 1(e)].

As expected, the effects of a second phase on the Poisson's ratio were amplified for higher values of $\frac{E_{h}}{E_{s}}$ [Figs. 2(a)-2(d)]. The same held for the fraction of the hard phase [Figs. 2(a)-2(d)]. Stress and strain localizations

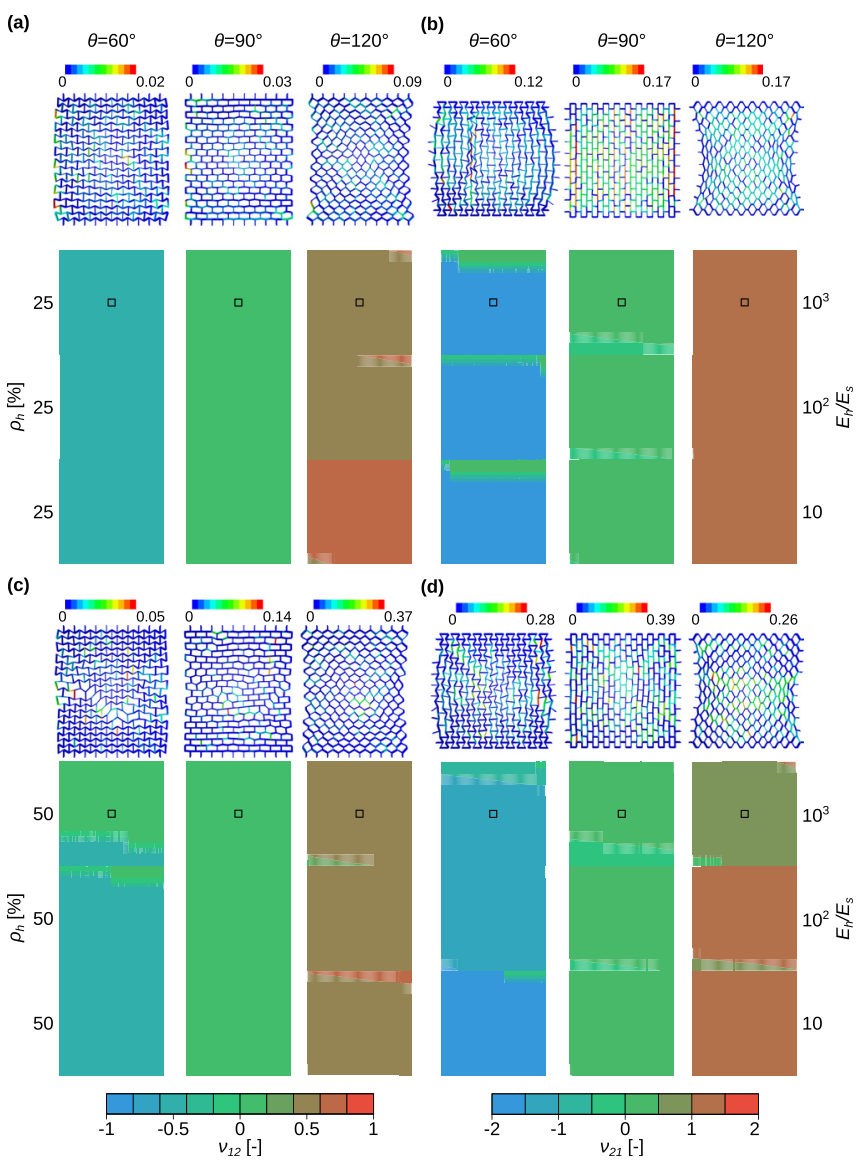

FIG. 2. The Poisson's ratios of the random multi-material lattice structures made with three unit cell geometries, three values of $\frac{E_{h}}{E_{s}}$, and two fractions of the hard material $\left(\rho_{h}\right)$. The values were calculated for directions 1 (a) and (c) and 2 (b) and (d). The strain distributions presented at the top of the Poisson's ratio contours show the principal strains obtained from the numerical simulations at $10 \%$ applied strain and for the data points indicated with boxes.

were clearly present in the case of multi-material designs [Figs. 2(a)-2(d)]. In addition, the stress and strain localizations were clearly intensified in the case of a higher fraction of the hard phase (i.e., $\rho_{h}=50 \%$ ) [Figs. 2(a)-2(d)]. Assignig a hard phase to a random place within a soft lattice structure could also affect the global deformation of the lattice structure and lead to an asymmetric deformation pattern (Fig. 2), as the harder elements of the lattice structure deflect or block the deformation flow.

In addition to randomly assigning the second (i.e., hard) phase material to a number of the elements of the lattice structure, we rationally designed the placement of the hard phase such that the elements to which the hard phase were assigned collectively created either a re-entrant (i.e., auxetic) or honeycomb (i.e., conventional) shape (Fig. 3). All designs were both $3 \mathrm{D}$ printed and analyzed using our computational models. Such rational placements of the second phase resulted in drastic changes in the elastic properties of the mechanical metamaterials [Figs. 3(a) and 3(b)]. For example, when we started from an auxetic lattice structure (legend: $\triangle$ ) and assigned the hard phase to specific elements of the structure in such a way that they created a global re-entrant shape, the Poisson's ratio remained largely unchanged while the stiffness was increased by $>10$ times in direction 1 (Fig. 3). Choosing a honeycomb shape for the hard elements assigned 
(a)

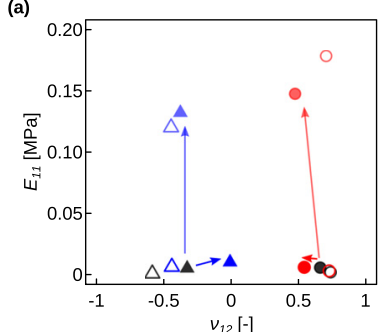

(c)
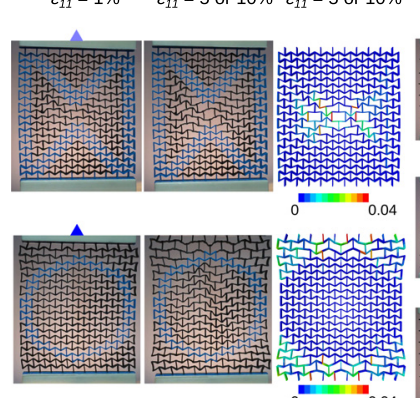

(d) $\varepsilon_{22}=1 \%$
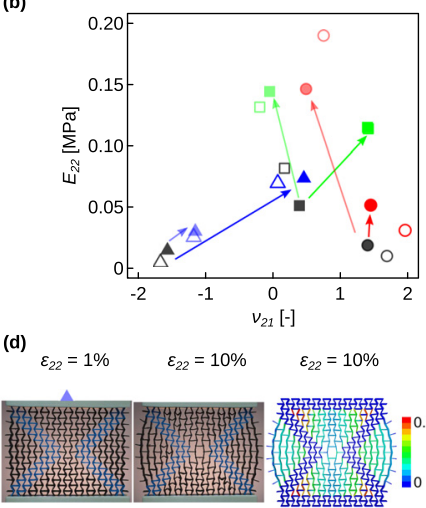

$\varepsilon_{22}=10 \%$
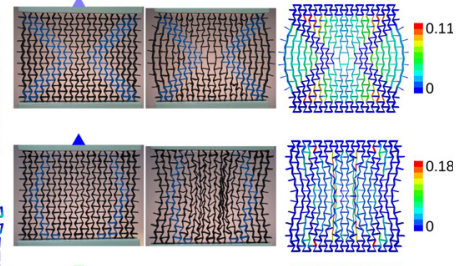

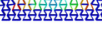
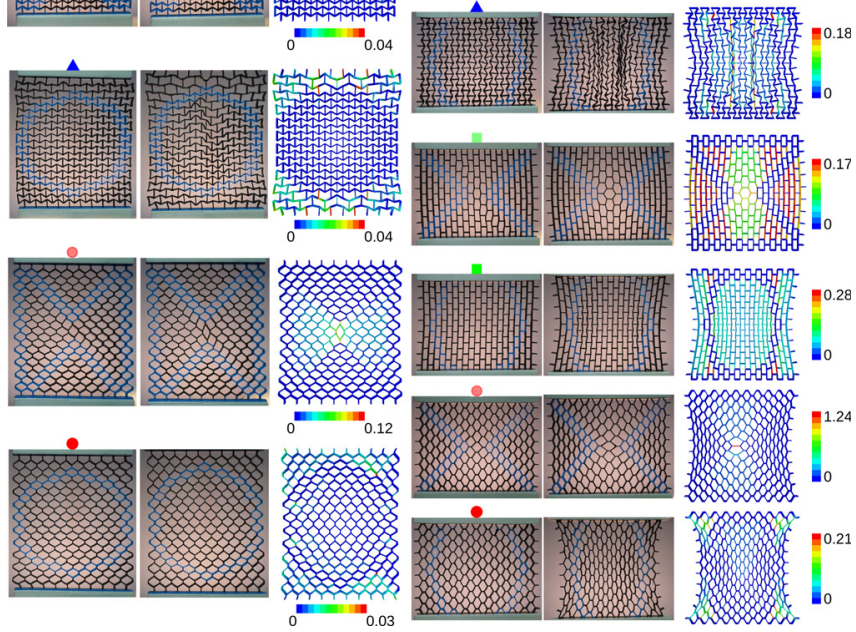

*For the first and third row FE results are shown at $\varepsilon_{11}=5 \%$

FIG. 3. The numerical (hollow markers) and experimental (solid markers) results for the elastic properties of multi-material lattice structures with rationally designed hard phases and tested in directions 1 (a) and 2 (b). The arrows compare the results of a corresponding lattice structure with a single soft material with those of the multi-material designs. The experimental and numerical deformation patterns are also compared with each other in directions 1 (c) and 2 (d). The strain distributions show the principal strains obtained using the computational models.

to the same type of the lattice structure (i.e., auxetic, legend: O) pushed the Poisson's ratio towards 0 , while not significantly changing the stiffness in direction 1 and substantially increasing the stiffness in direction 2 (Fig. 3). Various types of drastic evolutions in the stiffness and Poisson's ratio in both directions were observed for the other types of lattice structures and using different types of designs of the hard phase elements [see the arrows in Figs. 3(a) and 3(b)].

Interestingly, in the case of the lattice structures with a zero Poisson's ratio (legend: $\square$ ), introducing hard unit cells with a global re-entrant or honeycomb shape resulted in lattice structures with relatively large (negative or positive) values of the Poisson's ratio (Fig. 3). This shows that, in addition to the geometrical design, one could benefit from the spatial distribution of the mechanical properties as an effective tool to independently tailor the elastic properties of mechanical metamaterials.

Here, we assumed a constant thickness for the beam elements of the lattice structures and increased the rigidity of each beam only by increasing individual stiffnesses. Since the rigidity of each beam in these lattice structures is defined as $E I$, where $E$ is the elastic stiffness and $I$ is the second moment of inertia one could increase beam's rigidity by changing the second moment of inertia. In this case, it means using various beam thicknesses for each element. This is again mapping the elastic properties by manipulating the geometrical features of the lattice structures, although there is a limitation on the maximum thickness considered for such designs. We also used the dual-material for the construction of these metamaterials. However, different hardsoft elastic ratios or even a gradient in the mechanical properties can be taken into account for the construction of these materials.

The results of this study clearly show that both random and rational distributions of a hard phase could be used for independent tailoring of the elastic modulus and Poisson's ratio of a soft mechanical metamaterial. The spatial distribution of the mechanical properties could also be used for independent tailoring of the elastic properties in different directions, thereby allowing for the rational design of anisotropic mechanical metamaterials. Our computational models clearly show that, at the micro-scale, nonhomogeneous strain distributions and localized deformations are responsible for the observed behavior on the macro-scale including not only the effective elastic properties but also such phenomena as asymmetric deformations. From the mechanistic viewpoint, the assignment of a hard phase to random elements within the lattice structure locally deflects the flow of deformation towards these hard elements, thereby disrupting the uniform deformation flow resulting from the geometrical design of the lattice structure. As the stiffness of the hard phase or its fraction increases, this effect will be stronger. For lattice structures whose Poisson's ratio is already largely positive or negative, this random disruption of the deformation is unlikely to increase the Poisson's ratio further, which is why the absolute value of the Poisson's ratio tends to decrease when a hard phase is randomly assigned to lattice structure with $\theta=60^{\circ}$ or $\theta=120^{\circ}$. In the case of $\theta=90^{\circ}$, the Poisson's ratio is already perfectly zero, meaning that any random defelctions of the deformation flow will likely increase the Poisson's ratio. However, there is an equal chance that this random disruption of the deformation flow results in a positive or negative Poisson's ratio. That explains the more or less symmetric region of the elastic modulusPoisson's ratio covered by random multi-material lattice strucutres with $\theta=90^{\circ}$. When the assignment of the hard phase is rationally designed (e.g., in the global shape of a reentrant structure of a honeycomb), very big jumps in the values of the Poisson's ratio are possible. Regarding the elastic modulus, addition of a hard phase clearly increases the resistance to deformation, thereby resulting in a higher elastic modulus. The level of the increase in the elastic modulus is, however, dependent on how effectively the hard phase is used for increasing the load-bearing capacity of the lattice strucutre in a specific direction. Adjusting the effectiveness of the hard phase in a specific direction could therefore be used for tuning the elastic modulus of the lattice strucutre with the upper bound given by the most efficient distribution of the hard phase. Together, all the above-mentioned mechanisms allow for independent tailoring of the elastic modulus and Poisson's ratio of the lattice strucutre regardless of the geometrical design. Keeping the geometrical design intact might be particularly useful when designing multi-physics 
metamaterials for which the geometrical design also determines other physical properties of the material (e.g., thermal expansion or mass transport properties).

Here, we showed that the spatial distribution of materials with different mechanical properties (i.e., soft and hard phases) could be used to tailor the elastic properties of cellular structures. However, the trade-off for the use of such combinations is the creation of local stress concentrations in the resulting lattices. The level of the stress concentrations can significantly increase with a higher mismatch between the hard and soft phases. Moreover, the presence of these stress riser regions can result in the formation of local buckling or local damage. Those can eventually facilitate crack initiation and propagation in the cellular structure, leading to pre-mature failure particularly under repetetive loading. One way to address this challenge would be to introduce intermediate phases with specific gradients in their mechanical properties to decrease the severity of the stress concetnrations.

Here, we performed monotonic quasi-static tensile tests, as we were primarily interested in the elastic properties of these structures. Systematic study of the failure and fatigue mechanisms would, however, require extensive fatigue tests that are suggested to be pursued in future research.

See supplementary material for the design parameters of lattice structures, image analysis steps, and strain evolution.

The authors declare no competing interests.

\footnotetext{
${ }^{1}$ A. Alderson and K. Alderson, "Auxetic materials," Proc. Inst. Mech. Eng., Part G 221(4), 565-575 (2007).

${ }^{2}$ K. E. Evans and A. Alderson, "Auxetic materials: Functional materials and structures from lateral thinking!," Adv. Mater. 12(9), 617-628 (2000).

${ }^{3}$ X. Ren, R. Das, P. Tran, T. D. Ngo, and Y. M. Xie, "Auxetic metamaterials and structures: A review," Smart Mater. Struct. 27(2), 023001 (2018).

${ }^{4}$ R. Lakes, "Negative Poisson's ratio materials: Response," Science 238, 551 (1987).
}

${ }^{5}$ H. Yasuda and J. Yang, "Reentrant origami-based metamaterials with negative Poisson's ratio and bistability," Phys. Rev. Lett. 114(18), 185502 (2015).

${ }^{6}$ M. Mirzaali, S. Janbaz, M. Strano, L. Vergani, and A. Zadpoor, "Shapematching soft mechanical metamaterials," Sci. Rep. 8(1), 965 (2018).

${ }^{7}$ Z. G. Nicolaou and A. E. Motter, "Mechanical metamaterials with negative compressibility transitions," Nat. Mater. 11(7), 608-613 (2012).

${ }^{8}$ K. Dudek, R. Gatt, M. Dudek, and J. Grima, "Negative and positive stiffness in auxetic magneto-mechanical metamaterials," Proc. R. Soc. A 474(2215), 20180003 (2018).

${ }^{9}$ M. Mirzaali, M. Habibi, S. Janbaz, L. Vergani, and A. Zadpoor, "Crumpling-based soft metamaterials: The effects of sheet pore size and porosity," Sci. Rep. 7(1), 13028 (2017).

${ }^{10}$ Q. Wang, J. A. Jackson, Q. Ge, J. B. Hopkins, C. M. Spadaccini, and N. X. Fang, "Lightweight mechanical metamaterials with tunable negative thermal expansion," Phys. Rev. Lett. 117(17), 175901 (2016).

${ }^{11} \mathrm{D}$. Chen and X. Zheng, "Multi-material additive manufacturing of metamaterials with giant, tailorable negative Poisson's ratios," Sci. Rep. 8, 9139 (2018).

${ }^{12}$ P. Vogiatzis, S. Chen, X. Wang, T. Li, and L. Wang, "Topology optimization of multi-material negative Poisson's ratio metamaterials using a reconciled level set method," Comput.-Aided Des. 83, 15-32 (2017).

${ }^{13}$ Y. Wang, J. Gao, Z. Luo, T. Brown, and N. Zhang, "Level-set topology optimization for multimaterial and multifunctional mechanical metamaterials," Eng. Optim. 49(1), 22-42 (2017).

${ }^{14}$ S. Janbaz, M. McGuinness, and A. A. Zadpoor, "Multimaterial control of instability in soft mechanical metamaterials," Phys. Rev. Appl. 9(6), 064013 (2018).

${ }^{15}$ M. Mirzaali, M. Edens, A. H. de la Nava, S. Janbaz, P. Vena, E. Doubrovski, and A. Zadpoor, "Length-scale dependency of biomimetic hard-soft composites," Sci. Rep. 8(1), 12052 (2018).

${ }^{16}$ K. Wang, Y.-H. Chang, Y. Chen, C. Zhang, and B. Wang, "Designable dual-material auxetic metamaterials using three-dimensional printing," Mater. Des. 67, 159-164 (2015).

${ }^{17}$ M. Mirzaali, R. Hedayati, P. Vena, L. Vergani, M. Strano, and A. Zadpoor, "Rational design of soft mechanical metamaterials: Independent tailoring of elastic properties with randomness," Appl. Phys. Lett. 111(5), 051903 (2017).

${ }^{18}$ H. M. Kolken, S. Janbaz, S. M. Leeflang, K. Lietaert, H. H. Weinans, and A. A. Zadpoor, "Rationally designed meta-implants: A combination of auxetic and conventional meta-biomaterials," Mater. Horiz. 5(1), 28-35 (2018).

${ }^{19}$ R. Hedayati, M. Mirzaali, L. Vergani, and A. Zadpoor, "Action-at-a-distance metamaterials: Distributed local actuation through far-field global forces," APL Mater. 6(3), 036101 (2018).

${ }^{20}$ I. Masters and K. Evans, "Models for the elastic deformation of honeycombs," Compos. Struct. 35(4), 403-422 (1996). 